\title{
Magnetic resonance spectroscopic imaging of brain injury after nasopharyngeal cancer radiation in early delayed reaction
}

\author{
W.-S. Chen ${ }^{1}$, J.-J. Li ${ }^{1}$, J.-H. Zhang' ${ }^{2}$ L. Hong ${ }^{1}$, Z.-B. Xing ${ }^{1}$, \\ F. Wang ${ }^{1}$ and C.-Q. Li ${ }^{1}$ \\ ${ }^{1}$ Department of Radiology, People's Hospital of Hainan Province, \\ Haikou, China \\ ${ }^{2}$ Department of Radiology, East Hospital, Tongji University School of Medicine, \\ Shanghai, China \\ Corresponding authors: J.-J. Li / J.-H. Zhang \\ E-mail: jianjunlicn@yeah.net / cjr.lijianjun@vip.163.com
}

Genet. Mol. Res. 13 (3): 6848-6854 (2014)

Received June 27, 2013

Accepted January 13, 2014

Published August 29, 2014

DOI http://dx.doi.org/10.4238/2014.August.29.6

\begin{abstract}
This study aimed to investigate the value of magnetic resonance spectroscopy (MRS) imaging in assessing nasopharyngeal carcinoma radiotherapy during the early delayed reaction period. Eighty cases of nasopharyngeal cancer treated with radiotherapy within the same period underwent MRS imaging before or after radiotherapy. Of the 80 cases, 47 underwent MRS imaging on the 3rd, 4th, 6th, and 12th months after radiotherapy. The trends of the primary metabolite concentration at different time points were monitored and compared with the corresponding data after radiotherapy. Repeated measures analysis of variance was performed. At the end of radiotherapy, the $\mathrm{N}$-acetyl aspartate (NAA)/creatine (Cr), choline (Cho)/Cr, and NAA/ Cho ratios were reduced to the lowest levels after 3 months. However, increasing trends were observed from the 4th to the 12th month. On the 12th month, stable levels were reached with statistically significant differences $(\mathrm{F}=316.02,53.84,286.68 ; \mathrm{P}<0.01)$. MRS reflected the
\end{abstract}


radiation injury-repair process in the brain of a nasopharyngeal cancer patient during early delayed reaction. This non-invasive monitoring of changes in brain tissue metabolite concentrations provides valuable information for prognosis.

Key words: Nasopharyngeal tumor; Radiotherapy; Brain injury; Prognosis; Magnetic resonance spectroscopy

\section{INTRODUCTION}

Nasopharyngeal carcinoma is the most commonly occurring head and neck tumor in southern China, characterized by an increasing incidence trend. Radiotherapy has been the preferred treatment for nasopharyngeal cancer; however, the method is not free from side effects, with temporal lobe injury being the most common complication (Tian et al., 2002). Magnetic resonance spectroscopy (MRS) has provided a new assessment method for the study of brain radiation injury. Previous research has mainly focused on the metabolic changes during radiotherapy (hyperacute period) (Vikhoff-Baaz, 2001; Kaminaga and Shirai, 2005; Song et al., 2007). The early delayed reaction period after radiotherapy has been mentioned in a few reports (Chen et al., 2007; Qiu et al., 2007; Sundgren et al., 2009). However, brain injury induced by radiotherapy for nasopharyngeal cancer is relatively slow and is reversible within a certain range. In particular, the degree of damage repair during the early delayed reaction period is decisively significant in the final prognosis. Therefore, monitoring the trend of metabolic changes in the brain tissue during the early delayed reaction phase can provide important information for the outcome of radiation-induced brain injury (Perry and Schmidt, 2006). In the present study, a dynamic follow-up was carried out for patients with nasopharyngeal cancer in the early delayed reaction period by MRS. The parameters before and after radiotherapy were compared to explore the possible repair mechanisms and outcomes of brain radiation injury after radiotherapy for nasopharyngeal cancer.

\section{MATERIAL AND METHODS}

\section{Case and inclusion criteria}

From July 2006 to December 2011, 80 patients of the Hainan Provincial People's Hospital were initially diagnosed with nasopharyngeal cancer through imaging examination and nasopharyngeal lesion biopsy (excluding other brain and systemic diseases). Conventional magnetic resonance imaging (MRI) and MRS imaging were performed before radiotherapy and at the radiotherapy doses of 20, 40, and $60 \mathrm{~Gy}$. Of the 80 patients, 47 underwent MRS follow-up 6 and 12 months after radiotherapy (the other 33 cases were excluded from this group owing to the long follow-up interval time or loss to follow-up, including 36 males and 11 females, aged 37 to 63 years old. The average age was $46 \pm 4$ years old. Radiation therapy involved the face-neck joint portal, nasopharynx, and neck. The lower part of the bilateral temporal lobe was included in the radiation field. The conventional fractionation method was 2 Gy/day, 5 times a week. This study was conducted in accordance with the Declaration of Helsinki and with approval from the Ethics Committee of People's Hospital of Hainan Province. Written informed consent was obtained from all participants. 


\section{Spectral acquisition methods}

MRI was implemented using a GE Signa 1.5 T MR scanner. The conventional sequence included an axial plane T2WI (TR $4000 \mathrm{~ms}$, TE $120 \mathrm{~ms}$ ), T1WI (TR $400 \mathrm{~ms}$, TE $15 \mathrm{~ms}$ ), and fluid-attenuated inversion recovery (FLAIR) T2WI sequence (TR $6000 \mathrm{~ms}$, TE $120 \mathrm{~ms}$, TI $2000 \mathrm{~ms}$ ). FLAIR-T2WI was regarded as the position figure for spectral acquisition, whereas point-resolved spectroscopy (PRESS) and voxel acquisition (TR $1000 \mathrm{~ms}$, TE $144 \mathrm{~ms}$ ) were also implemented. Each spectral scan range was kept constant (with reference to the scan location map from the first time). The bilateral anterior temporal lobe and 6 saturated zones were set. Automatic shimming reached the full width at half maximum of water at 6 to 12 after water suppression $>98 \%$. Acquisition was automatically performed.

\section{Spectral analysis}

The data collected were sent to the workstation with ADW 4.0. The Functool 2 software was used in post-processing and analysis. The small voxel within spectral scanning with the size of about $30 \pm 5 \mathrm{~mm}^{2}$ was automatically analyzed by the software. In the bilateral temporal lobe, 2 small voxels with the highest signal-to-noise ratios and ideal spectral curves were selected for analysis. The bilateral concentration ratios of the major metabolites, $\mathrm{N}$-acetyl aspartate (NAA), creatine ( $\mathrm{Cr}$ ), and choline (Cho), and the average of NAA/Cr, $\mathrm{Cho} / \mathrm{Cr}$, and $\mathrm{Cho} / \mathrm{NAA}$ were determined to analyze the change trends of the metabolite concentration ratios during the early period of delayed reaction.

\section{Statistical analysis}

The LC Model analysis software was adopted for statistical analysis to overcome $\mathrm{Cr}$ content fluctuations caused by radiation. Spectral data are reported as means $\pm \mathrm{SD}$. The data before radiotherapy were regarded as covariates. At different time points during the early delayed reaction phase, the NAA/Cr, $\mathrm{Cho} / \mathrm{Cr}$, and NAA/Cho values were subjected to repeated measures analysis of variance. $\mathrm{P}<0.05$ was considered to be statistically significant.

\section{RESULTS}

Of the 80 nasopharyngeal cancer cases with initial radiotherapy, 47 cases underwent MRS follow-up up to the 12th month after therapy. During this period, the use of neurotrophic drugs, vasodilator, and hormones were not allowed. Eleven patients experienced thirst, burning sensation in the oral mucosa, dizziness, and other mild reactions during radiotherapy. Eventually, all those manifestations disappeared. One month after radiotherapy, 19 cases of slight headache and dizziness lasting several days to 2 weeks were reported.

The trends of the concentration ratios of the 3 main metabolites were also described. 1) Compared with those before radiotherapy, $\mathrm{NAA} / \mathrm{Cr}, \mathrm{Cho} / \mathrm{Cr}$, and $\mathrm{NAA} / \mathrm{Cho}$ values were reduced after radiotherapy. 2) Three months after radiotherapy, the 3 ratios were further reduced, and the amplitude was less than that during the radiation process. 3) Increasing trends were observed from the 4 th to the 12th month after radiotherapy. On the 12th month, the 3 ratios were higher than those after radiotherapy to varying degrees. The $\mathrm{Cho} / \mathrm{Cr}$ and NAA/ 
Cho values were close to but still lower than the pre-radiotherapy values. Compared with the data after radiotherapy, the differences were statistically significant (Table 1). 4) Three months after radiotherapy, 4 cases showed inverted lactate (Lac) peaks, which lasted 1 to 3 months before gradually disappearing.

\begin{tabular}{l} 
Table 1. Comparisons among the 3 main metabolites ratios of 47 in 80 cases from the pre-radiotherapy to after \\
radiotherapy 12 months. \\
\hline Time \\
\hline Before radiotherapy
\end{tabular}

Three ratios in the 12th and the 3rd month after radiotherapy had significant differences $(\mathrm{P}<0.01)$, the ratios in the 12th month after radiotherapy compared with these before radiotherapy the differences were not significant statistically $(\mathrm{P}>0.05)$.

Increases in $\mathrm{NAA} / \mathrm{Cr}$ of $2.12,20.86$, and $24.46 \%$ were observed on the 4 th, 6 th, and 12 th months, respectively. The increased percentages for $\mathrm{Cho} / \mathrm{Cr}$ were $2.36,8.66$, and $13.39 \%$, respectively, whereas those for NAA/Cho were $1.96,25.4$, and $30.39 \%$, respectively.

\section{DISCUSSION}

The cure dose of radiation therapy induces the risk of damage to normal tissue surrounding the radiation field. The effect of radiotherapy positively correlates with the radiation dose. Increasing the dose also increases the risk of normal brain tissue damage. Radioactive brain damage was found to occur in 5 to $24 \%$ of cases (Perry and Schmidt, 2006). Radiation acts on brain tissues, causing a series of physiological and biochemical reactions, which result in varying degrees of dysfunction and changes in cell structure. The mechanism of radiation damage is very complex, and no universally accepted argument has yet been established. Generally, the main mechanism of injury includes several aspects, such as nerve and glial cell damage caused by direct radiation, as well as the demyelination, softening, and necrosis of white matter. Oxidative radicals damage the cell membrane, causing cell dysfunction, fibrosis, stenosis, and the occlusion of small- and medium-sized arteries, as well as a reduced arterial supply, which induces hypoxia (Armstrong et al., 2004). Radiation also causes autoimmune reactions. Based on the onset time and clinical symptoms, brain radiation injury is mainly divided into acute reaction period, early delayed reaction phase, and late delayed reaction period (Hong and Guo, 2003). Brain radiation injury can be repaired at a certain stage and to a certain extent. Conventional computed tomography and MRI can only detect severe and irreversible damage during the late delayed reaction phase, whereas MRS can monitor damage at the molecular level by measuring the metabolic concentration trends during the early delayed reaction period (Haun et al., 2010). Thus, MRS is very important in the assessment of repair mechanisms and possible outcomes of radiation-induced brain injury.

The changes in NAA/Cr and its possible causes in the early delayed reaction period 
were explored. NAA is a marker of neuron structure and function. The number of neurons per unit volume of brain tissue and the accompanying metabolite changes can alter the NAA/Cr values (Tong et al., 2004). The results of the present study showed that NAA/Cr decreased during radiotherapy down to minimum levels 3 months after radiotherapy. The ionizing effects of radiation include the destruction of the cell membrane of the neuronal mitochondria, or the stimulation of the mitochondria to produce more free radicals (Fan and Sun, 2005). Impaired mitochondrial membrane function reduces energy metabolism, thereby decreasing the NAA/ $\mathrm{Cr}$ ratio. At this stage, the radiation dose used was small, and only a small number of neurons underwent apoptosis. A decreased NAA/Cr value is mainly caused by neuronal metabolic disorders occurring without destruction of the cell structure (Song et al., 2006). The direct effects of radiation disappeared within 3 months after radiotherapy. However, because of the time sequential characteristic of ionizing radiation injury, neuronal damage does not end with the immediate cessation of radiation treatment. In addition, the injury mechanism becomes more complicated with the aggravation of injury. This observation can be attributed to the synergistic effect of one or more injuries. For example, radiation can lead to hyaline degeneration in the blood vessel wall, intima reactive hyperplasia, vascular permeability (Miller, 2004), increased number of water molecules between the extravascular cells, and reduced neuronal population in every voxel unit. The synergy of these factors causes the NAA/Cr ratio to drop to its lowest level 3 months after radiotherapy.

The radiation damage of neurons is reversible within a certain range and to a certain extent. As the results of the follow-up showed, 12 months after radiotherapy, the NAA/Cr values increased to varying degrees, but at generally lower values than pre-radiotherapy levels. Chen et al. (2007) reported similar results. In our analysis, most neuronal dysbolism and dysfunction were periodical during and 3 months after radiotherapy. No structural damage and cell death was observed. Thus, the gradual recovery of metabolism and function of most damaged neurons was evident a few months after the termination of radiation. Meanwhile, cerebral vascular injury repaired slowly, permeability returned to near normal, the leaking water molecules between cells were absorbed, and the number and density of neurons increased in every volume unit. Consequently, the NAA/Cr value indicated an upward trend, but did not recover to pre-radiotherapy levels. This observation can be attributed to the small extent of neuronal apoptosis or irreversible fatal injuries that occurred (Wilding et al., 2006).

The changes in the $\mathrm{Cho} / \mathrm{Cr}$ value and the possible mechanism involved during the early delayed response period are described as follows. Cho indicates the total choline content, which includes 3 kinds of cholines (phosphatidylcholine, phosphoric acid, and phosphoglycerol). It is related to myelin sheath formation and phospholipid metabolism in the cytomembrane. The Cho content in glial cells of brain tissue is significantly higher than that in neurons (Vikhoff-Baaz et al., 2001). Therefore, Cho levels in brain tissue depend mainly on the rates of glial cell membrane synthesis and degradation. The data showed that $\mathrm{Cho} / \mathrm{Cr}$ values decreased during radiotherapy, lasting up to 3 months after radiotherapy. Twelve months postradiotherapy, the values recovered to pre-radiotherapy levels. Radiation acts on glial cells, decreasing cellular activity, weakening metabolism, slowing down the phospholipid synthesis of the cytomembrane and myelin sheath formation, and decreasing Cho expression along with the $\mathrm{Cho} / \mathrm{Cr}$ value. After termination of radiation for a certain period, $\mathrm{Cho} / \mathrm{Cr}$ starts to rise, which can be attributed to the gradual restoration of energy metabolism. During radiotherapy, the disintegrating portion of myelin is absorbed, the swelling glial cells are gradually 
restored, and the density of glial cells increases. After radiotherapy, the proliferation of glial cells may take place, which increases the $\mathrm{Cho} / \mathrm{Cr}$ value to some extent. The results of these factors showed that Cho expression recovered to pre-radiotherapy levels. Kaminaga and Shirai (2005) found that after glioma surgery, a rise in the $\mathrm{Cho/Cr}$ value during radiotherapy was observed along with a decline in the delayed reaction trend. Ultimately, the value increases to near pre-radiotherapy levels. Some scholars believe that the radiotherapy-induced elevation of the $\mathrm{Cho} / \mathrm{Cr}$ value is associated with increased water-soluble phosphorylcholine, which is caused by tissue necrosis rooted during the early structural damage of brain cells (Plotkin et al., 2004). Some scholars have suggested that the rise in the $\mathrm{Cho} / \mathrm{Cr}$ value is related to the cytomembrane synthesis of glial cells. Song et al. $(2006,2007)$ found that the $\mathrm{Cho} / \mathrm{Cr}$ value decreased during radiotherapy, and that the ionizing effects of radiation are the primary factor affecting the metabolism and function of the cells. The subjects in this study received conventional fractionated radiotherapies ( 2 Gy/day, 5 times a week, with 2 intermittent days between every 10 Gy accumulated dose). The brain tissue only received edge radiation, and the possibility of necrosis was small. The Cho/Cr value initially decreased before it subsequently increased. Other similar reports state that after a small dose of radiotherapy, the metabolism of glial cells decreases and the $\mathrm{Cho} / \mathrm{Cr}$ value eventually increases to pre-radiation levels. The large extent of glial cell damage caused by nasopharyngeal radiotherapy on the edge of the temporal lobe is reversible. The trend of the $\mathrm{Cho} / \mathrm{Cr}$ value during radiotherapy and during the early delayed reaction varies with the radiotherapy mode. The mechanisms of glial cell injury vary with different modes and degrees of radiation (Chen et al., 2011). In addition, for a few cases in the group, the lactate peaked on the 3rd month after radiotherapy, which was caused by anoxia. This peak disappeared after a sustained period. Individual differences could exist in relatively serious vascular injuries because of insufficient local oxygen supply in cellular water molecules. The lactate peak gradually disappeared with the absorption of extracellular liquid. Generally, the $\mathrm{Cr}$ concentrations in brain metabolites are stabilized, which is usually regarded as an internal reference, but may decrease during brain radiation injury. The present study used the LC Model analysis software to overcome the statistical errors caused by denominator changes, thereby avoiding the impacts of NAA/Cr and Cho/Cr values induced by the fluctuations in creatine content.

However, limitations to this study are inevitable. Various factors, such as the skull, sphenoid sinus, and cerebrospinal fluid, interfered with the spectroscopic imaging of the temporal lobe. Sometimes, repeated adjustment of the scanning area and the saturated zone was needed to achieve the acquisition requirements. Guaranteeing the same exact positions of the small voxel selected from the same patient at each time for analysis was a challenge. Averages obtained from larger sample sizes and a greater number of small voxels can reduce this bias. In addition, the majority of nasopharyngeal cancer cases exhibited the ideal short-term effects after radiotherapy (Lam et al., 2012). Compared with other diseases, follow-up study after radiotherapy was difficult, owing to some factors such as patient compliance. The long-term cyclical changes of the NAA/Cr and $\mathrm{Cho} / \mathrm{Cr}$ values thus need further follow-up studies.

In summary, neuronal damage exhibits functional and metabolic disorders after radiotherapy. These changes are reversible, but when accompanied by apoptosis or death of a few neurons during the early reaction period, the $\mathrm{NAA} / \mathrm{Cr}$ value can no longer recover to pre-radiotherapy levels (Chen et al., 2007). After injury, the $\mathrm{Cho} / \mathrm{Cr}$ value decreases with increasing glial cell membrane dysfunction. However, because of recovered membrane function and glial 
hyperplasia during the early delayed response phase, the value increases to near pre-radiotherapy levels. MRS monitors the changes in metabolite concentration during radiation-induced brain injury in the early delayed reaction period. Thus, MRS is beneficial in the assessment of the extent of repair and prognosis of radiation-induced brain damage.

\section{ACKNOWLEDGMENTS}

Research supported by the National Natural Science Foundation of China (\#81360233, \#81271389), the Hainan Key Science and Technology Project (\#ZDXM20130069), the Hainan Natural Science Foundation-funded project (\#812154), the Guangdong Natural Science Foundation-funded project (\#S2013010014545), and the Hainan Health Department research project approval (\#2011-23, \#2012PT-19).

\section{REFERENCES}

Armstrong CL, Gyato K, Awadalla AW, Lustig R, et al. (2004). A critical review of the clinical effects of therapeutic irradiation damage to the brain: the roots of controversy. Neuropsychol. Rev. 14: 65-86.

Chen WS, Li JJ, Xing ZB, Hong L, et al. (2011). 1H-MR spectroscopy study on radiation-induced injury of the temporal lobes in the elderly patients with nasopharyngeal carcinoma. Chin. J. Radiol. 45: 1023-1027.

Chen YB, Liu ZH, Bao DL and Pan JJ (2007). 1H proton magnetic resonance spectroscopy explore the radiation-induced early brain injury of nasopharyngeal carcinoma. J. Fujian Med. Univ. 41: 222-224.

Fan DS and Sun M (2005). Mitochondria and ionizing radiation damage relations research status. J. Modern Oncol. 13: 843-846.

Haun JB, Yoon TJ, Lee H and Weissleder R (2010). Magnetic nanoparticle biosensors. Wiley Interdiscip. Rev. Nanomed. Nanobiotechnol. 2: 291-304.

Hong MH and Guo X (2003). Nasopharyngeal Carcinoma. Chinese Medicine Science and Technology Press, Beijing, 255-257.

Kaminaga T and Shirai K (2005). Radiation-induced brain metabolic changes in the acute and early delayed phase detected with quantitative proton magnetic resonance spectroscopy. J. Comput. Assist. Tomogr. 29: 293-297.

Lam WW, Ye M and Fielding R (2012). Trajectories of quality of life among Chinese patients diagnosed with nasopharynegeal cancer. PLoS One 7: e44022.

Miller NR (2004). Radiation-induced optic neuropathy: still no treatment. Clin. Experiment. Ophthalmol. 32: 233-235.

Perry A and Schmidt RE (2006). Cancer therapy-associated CNS neuropathology: an update and review of the literature. Acta Neuropathol. 111: 197-212.

Plotkin M, Eisenacher J, Bruhn H, Wurm R, et al. (2004). 123I-IMT SPECT and 1H MR-spectroscopy at $3.0 \mathrm{~T}$ in the differential diagnosis of recurrent or residual gliomas: a comparative study. J. Neurooncol. 70: 49-58.

Qiu SJ, Zhang XL, Zhang Y and Jiang M (2007). Proton magnetic resonance spectroscopy for radiation encephalopathy induced by radiotherapy for nasopharyngeal carcinoma. Nan. Fang Yi Ke. Da. Xue Xue Bao 27: 241-246.

Song Q, Xia LM, Wang CY and Fang DY (2006). 1H-MRS study on radiation-induced injury of the brain in early acute reaction stage after nasopharyngeal carcinoma radiotherapy. Chin. J. Radiol. 40: 590-593.

Song Q, Xia LM, Wang CY and Feng DY (2007). MR spectroscopy and diffuse tensor imaging in super-acute stage of radiation induced brain injury. Radiol. Pract. 22: 687-690.

Sundgren PC, Nagesh V, Elias A, Tsien C, et al. (2009). Metabolic alterations: a biomarker for radiation-induced normal brain injury-an MR spectroscopy study. J. Magn. Reson. Imaging 29: 291-297.

Tian Y, Guo Z and Zhu M (2002). Radiation encephalopathy in nasopharyngeal carcinoma patients in mainland China: a systematic evaluation. Zhonghua Zhong. Liu Za Zhi. 24: 471-473.

Tong Z, Yamaki T, Harada K and Houkin K (2004). In vivo quantification of the metabolites in normal brain and brain tumors by proton MR spectroscopy using water as an internal standard. J. Magn. Reson. Imaging 22: 1017-1024.

Vikhoff-Baaz B, Malmgren K, Jonsson L, Starck G, et al. (2001). Lateralisation with magnetic resonance spectroscopic imaging in temporal lobe epilepsy: an evaluation of visual and region-of-interest analysis of metabolite concentration images. Neuroradiology 43: 721-727.

Wilding CS, Cadwell K, Tawn EJ, Relton CL, et al. (2006). Mitochondrial DNA mutations in individuals occupationally exposed to ionizing radiation. Radiat. Res. 165: 202-207. 